$$
\text { "szabo molnar" — 2004/7/22 — 15:23 — page } 103 \text { — \#1 }
$$

\title{
Applications of methods of descriptive geometry in solving ordinary geometric problems
}

\author{
JózSef Szabó and Ildikó Molnár \\ Dedicated to Professor Oswald Giering on his $70^{\text {th }}$ birthday
}

\begin{abstract}
The importance of descriptive geometry is well-known in two fields. Spatial objects can be mapped bijectively onto a plane and then we can make constructions concerning the spatial objects. The other significance of descriptive geometry is that mathematical visual perception of objects in three-dimensional space can be improved by the aid of it. The topic of this paper is an unusual application of descriptive geometry. We may come across many geometric problems in mathematical competitions, in entrance examinations and in exercise books whose solution is expected in a classical way, however, the solution can be found more easily and many times more general than it is by the standard manner. We demonstrate some of these problems to encourage to use this geometric method. Understanding the solution requires very little knowledge of descriptive geometry, however, finding a solution needs to have some idea of descriptive geometry.
\end{abstract}

Key words and phrases: geometry, descriptive geometry, didactics.

ZDM Subject Classification: G10, G80.

In this paper we omit other noteworthy constructive methods such as cyclography or very special descriptive geometric tasks. We select problems whose solution is very simple with the help of descriptive geometry. For example, in Vigassy's [8] exercise book for seminars there are about 100 problems which can be treated in that manner. Sometimes planar problems can be solved easily by 'interpretation in space'. Stiefel's [7] proof of Brianchon's Theorem is noteworthy. Also Jenő Horváth [1] published several tasks with elegant geometric solutions.

Copyright (c) 2004 by University of Debrecen
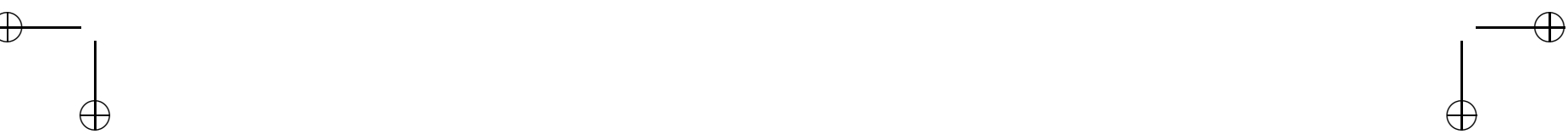
We demonstrate almost exclusively tasks that were set in competitions and the principle of the selection lies in the method used in their solutions. Acquiring the suitable method is obligatory in the training of teachers of descriptive geometry. In the paper no special knowledge of descriptive geometry is necessary, basics of front- and top-view projection are sufficient requirements (see [2], [4], [7]). The problems presented here are treated in the Constructive and Computer Geometry Course in the training of teachers of mathematics and descriptive geometry. But similar tasks often occur in the education of mathematics teachers not only in Hungary. The elegance of the geometric method usually causes excitement and keen interest.

At first we show a problem that gave the inspiration to apply descriptive geometry as a method for its solution. It was a task in the Students' Competition in Moscow in 1941 and it can be found in [6, task 4].

Problem 1. Let $e$ and $f$ be two skew lines perpendicular to each other. There is given a segment $R S$ greater than the distance of the lines. Let point $R$ move on $e$ and point $S$ on $f$. What is the locus of the midpoints $M$ of the segments $R S$ ?

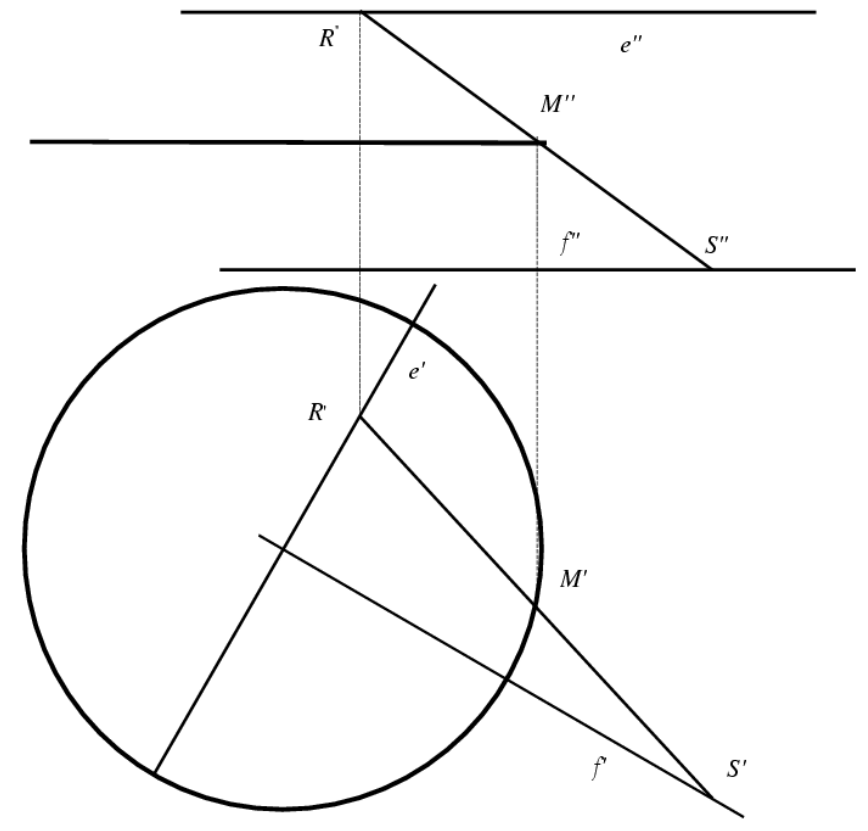


Solution. There exist planes that are parallel to both of the lines $e$ and $f$. Let the image-plane of the top-view be parallel to these planes. We have two lines perpendicular to each other in the top-view and two lines parallel to the axis of MonGE's top-front-view system. The projections of points $M$ are midpoints in both projections, too, because of the invariance of the ratio of segments under parallel projections.

We can state, by the aid of the front-view, that the locus is a planar figure, its front-view is on the line that is parallel to $e^{\prime \prime}$ and $f^{\prime \prime}$ and is at equal distance from both of them. The plane of the locus is parallel to the plane of the top-view, that is why we can see the locus in actual size from above. Since the distance of $e$ and $f$ and the length of the segment $R S$ is constant, the length of the segment $R^{\prime} S^{\prime}$ is constant, too. Since $M^{\prime}$ is the midpoint of the segment $R^{\prime} S^{\prime}$, the locus is a circle.

Generalization 1. (It was not included in the task.) If $P$ is another fixed point of the segment $R S$, then the locus is, again well-known, an ellipse. If $P$ divides the given segment into two pieces of length $a$ and $b$ then $P^{\prime}$ divides $R^{\prime} S^{\prime}$ into two segments of length $a^{\prime}$ and $b^{\prime}$ where $a: b=a^{\prime}: b^{\prime}$ (since parallel projections preserve the ratio of the length of segments). The co-ordinates of $P^{\prime}$ can be determined with the help of the top-view: $x=a^{\prime} \cdot \cos \alpha, y=b^{\prime} \cdot \sin \alpha$ and this results in the equation $\frac{x^{2}}{a^{\prime 2}}+\frac{y^{2}}{b^{\prime 2}}=1$.

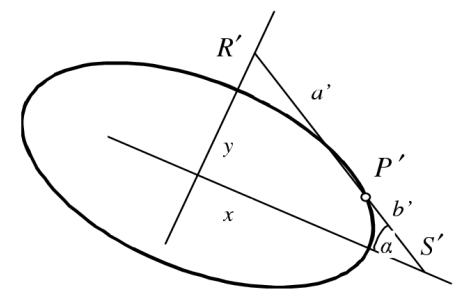

Generalization 2. Since we can see the actual angle of the lines from above, the question may arise what the locus will be if the given skew lines are not perpendicular to each other. We may think that the answer will be the affine image of the figure above since affinity preserves lines, ratio of signed distances of collinear points, and the affine image of an ellipse is an ellipse included the ones having equal axes, viz. the circle. If we proceed in this way we make a mistake, namely whereas the top-view of the moving segment has constant length as we 
have proved before, the affinity maps the segment $R^{\prime} S^{\prime}$ in the figure above onto a segment whose length is not constant.

In spite of this, the locus is an ellipse, the proof is the following:

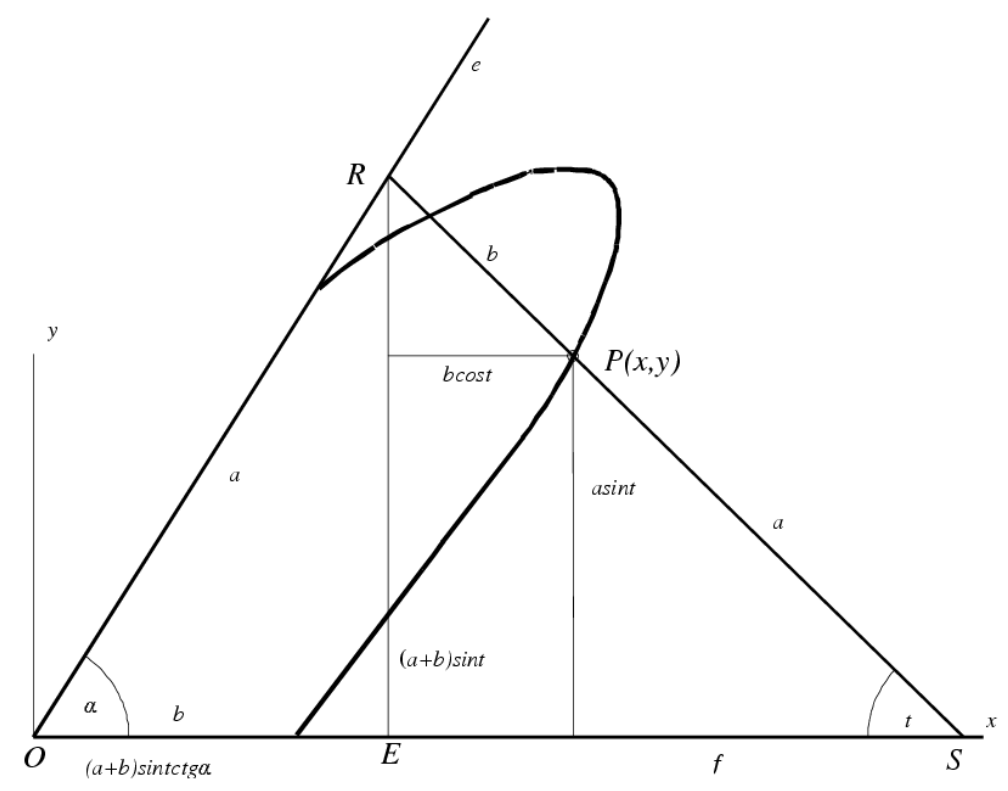

Let the two given lines intersect at an angle $\alpha$. Let the angle of the moving segment and the axis $x$ be $t$ and this will be the parameter. Then

$$
\begin{aligned}
& R E=(a+b) \sin t, \\
& O E=(a+b) \sin t \operatorname{ctg} \alpha .
\end{aligned}
$$

Hence we have

$$
\begin{aligned}
& x=(a+b) \operatorname{ctg} \alpha \sin t+b \cos t, \\
& y=a \sin t
\end{aligned}
$$

for the co-ordinates of $P$.

Let the constant $(a+b) \operatorname{ctg} \alpha$ be denoted by $q$ in the first co-ordinate, then we obtain the form

$$
x=q \sin t+b \cos t
$$


Now we eliminate parameter $t$.

$$
\begin{gathered}
\frac{y}{a}=\sin t \\
\frac{x-q \frac{y}{a}}{b}=\cos t \\
\left(\frac{y}{a}\right)^{2}+\left(\frac{x-q \frac{y}{a}}{b}\right)^{2}=\sin ^{2} t+\cos ^{2} t=1 \\
a^{2} x^{2}+\left(q^{2}+b^{2}\right) y^{2}-2 q a x y-a^{2} b^{2}=0
\end{gathered}
$$

This equation is that of the ellipse, since all of its points are finite and it is a non-degenerate quadratic curve.

If we know that $R^{\prime} S^{\prime}$ is constant then its one-parameter forced movement with endpoints on two straight lines is the well-known 'ellipse motion', which one applies in the socalled 'paper strip construction' of an ellipse with given conjugate diameter lines, then the analytic calculation is unnecessary.

We think the problem and the generalizations show the usefulness of descriptive geometry as a method.

The next task was worth the most points in a central entrance examination and it aimed at the application of geometrical mean.

Problem 2. Let us erect perpendiculars to the altitudes of an acute triangle $A B C$ at its orthocentre $M$ and construct the Thales' half-circles over the triangle's altitudes. On each perpendicular label each intersection point with the matching Thales half-circle by $R, S, T$. Prove that the segments $M R, M S, M T$ obtained in this way have equal length.

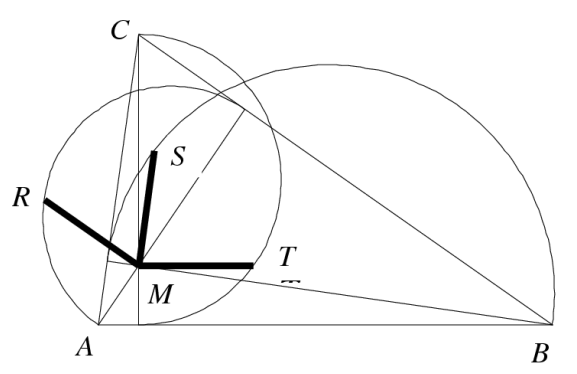


Solution. The three segments show the distance of the origin of the orthogonal axonometry from the image-plane; a point has only one distance from a plane. Thus we are ready.

Without being aware of this, we may say that three concurrent and pairwise perpendicular lines intersect a plane at the vertices of an acute triangle and the orthogonal projections of the lines onto the plane are the altitudes of this triangle. The distance of the common point of the lines and the plane can be determined by making a 'side-view' through one of the altitudes.

The intersection points of a circle and an ellipse cannot be determined by Eucleadian construction. Kárteszi [2] gives a method for this problem if they are concentric.

Problem 3. Determine the intersection points of a circle and an ellipse if they are concetric.

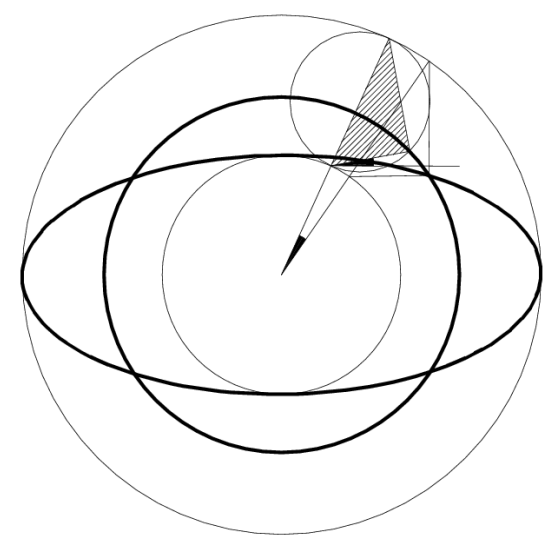

Solution. The solution is based on the construction of points of an ellipse with the help of concentric circles, called "DE LA HIRE's construction".

Let us draw the two vertex circles of the ellipse and a ray from its centre. This ray intersects the circles at two points. Draw a Thales' circle over these points according to the figure. If the the radius $\mathrm{r}$ of the given circle fulfills $b<r<a(a$, $b$ denote the half axes of the ellipse), then the Thales' circle intersects the given circle at two points symmetric to the ray. We obtain two right angled triangles, in the figure one of them is shaded. Let us rotate it by the black angle around 
the common centre of the ellipse and the vertex circles such that the legs of the triangle become parallel to the axes of the ellipse. The vertex at the right angle, moving on the given circle, becomes a point of the ellipse according to de la Hire's construction.

This method, based on a planar recipe, cannot be generalized. We suggest treating the problem by 'interpretation in space': The circle and the ellipse might be seen as images of two planar sections of a sphere. Since the solution of the previous task would be rather trivial by this method, we pose the problem a little bit more generally:

Problem 4. Let an ellipse and a circle whose centre is on the line of the minor axis of the ellipse be given. Determine the points of intersection of the ellipse and the circle.

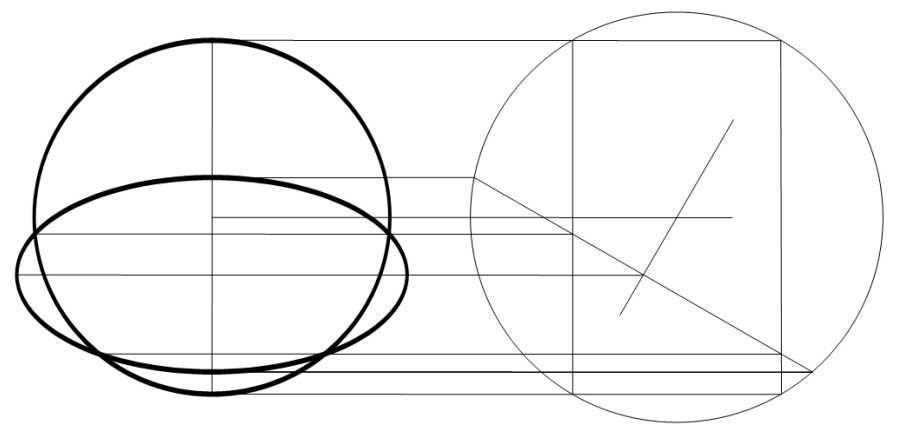

Solution. Now we consider the given figures as the top-view of two circles and construct their side-views. The ellipse is the top-view of a circle whose radius is the major axis. This circle is set in the space so that its top-view is the given ellipse with the minor axis. There exists a one-parameter set of spheres through this circle. The centres of these spheres are on the line that passes through the centre of the circle and is perpendicular to the plane of the circle. It can be seen in the side-view. The given circle is the projection of a spherical circle that is parallel to the top-view. There exists a pencil of spheres through that circle, too. As the given figure is symmetric, the two lines consisting of the centres of the spheres of both pencils are in the same plane and intersect in the centre of the sphere carrying both circles. The side-views of the circles are segments. The circular image comes from two congruent circles of the sphere in parallel planes. 
Each pair of the intersection points in question comes from the intersection of one of those circles with the one having the elliptic image.

The problem is slightly more difficult if the centre of the circle is on the major axis of the ellipse.

The next problem was a task in a competition of Hungarian pupils specialized in mathematics (pupils had ten maths lessons a week) in the second round in 1980 (see [3]).

PRoBlem 5. The orthogonal projections of a planar quadrilateral on both two planes that are perpendicular to each other are squares whose sides are two units long. What is the perimeter of the quadrilateral if one of its sides is $\sqrt{5}$ ?

Solution. The projections on the planes perpendicular to each other induces the consideration in Monge-projection, it is natural to treat the squares as frontand top-views.
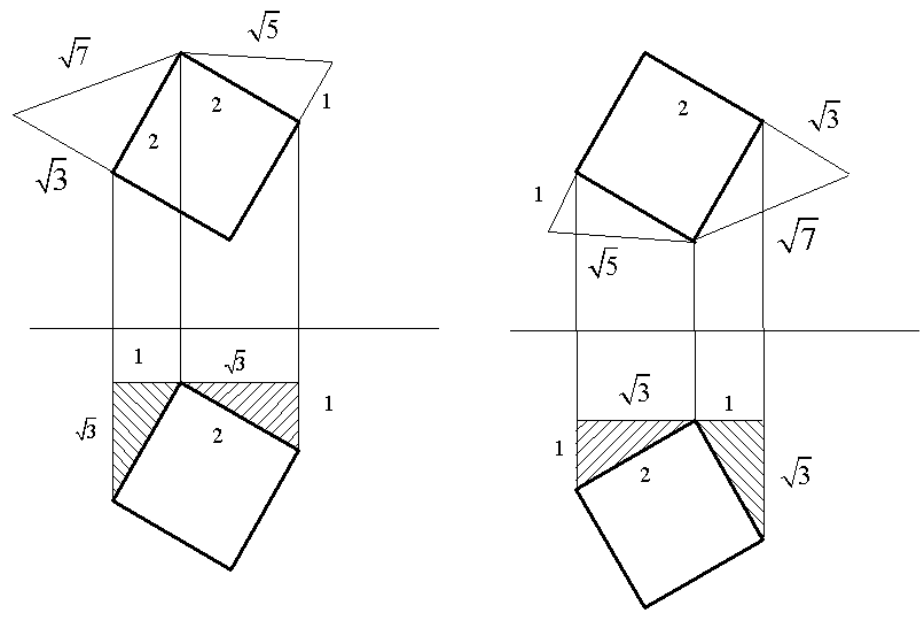

The term "planar" is unnecessary because it follows from the two given projections that the spatial figure is a parallelogram. Since the projections are congruent, they can be arranged as top- and front-views in two ways: they can be either translated into each other parallel to the order-lines or symmetric with the order-lines as symmetry rays. In each front-view we construct the triangles to determine the actual size from which we obtain the shaded triangles in the top-views. The difference of the order-lines provides that the other side of the 
parallelogram is $\sqrt{7}$ in both cases. Thus the perimeter of the quadrilateral is $p=2 \cdot(\sqrt{5}+\sqrt{7}))$ units.

The task does not ask for the area of the quadrilateral, but as it is an almost trivial conclusion, we pose the following problem:

Determine the area of the quadrilateral above:

As the parallelogram shows as congruent squares in both projections, its plane is parallel to one of the symmetry planes of Monge's image-planes. Thus, in both possible cases its slope angle to the first image-plane is $45^{\circ}$. Let $T$ denote the area of the quadrilateral and $T^{\prime}$ the area of its quadratic top-view, then by

$$
T^{\prime}=4=T \cos 45^{\circ}=T \frac{\sqrt{2}}{2}
$$

it follows that

$$
T=\frac{8}{\sqrt{2}}=8 \frac{\sqrt{2}}{2}=4 \sqrt{2} \text { sq units. }
$$

The following task was posed at an entrance exam at a university in 1960's, long before the central entrance examination system was established. Nobody solved it.

Problem 6. Let us rotate a unit cube around one of its diagonal and calculate the volume of the solid of revolution obtained in this way.
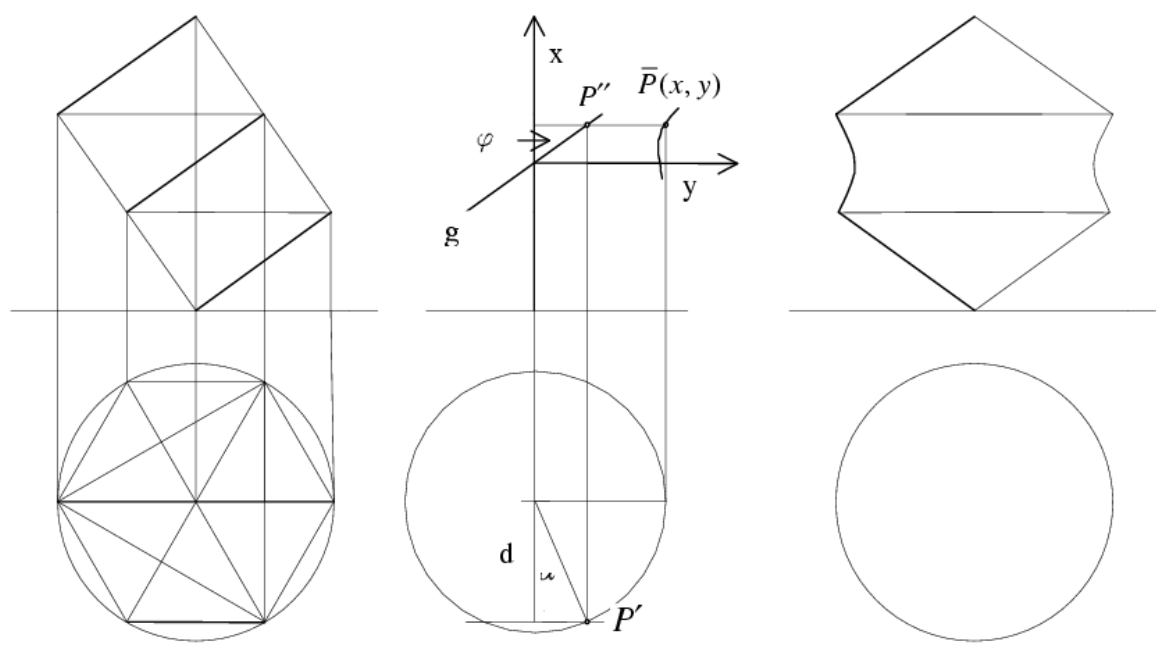
Solution. The planes on which the vertices are moving while the cube is being rotated divide the diagonal into three equal parts and they are perpendicular to the diagonal. Let the cube be placed into a Monge system of image-planes and let the diagonal be perpedicular to the plane of the top-view. Thus the contour of the top-view is a regular hexagon. Let us rotate the cube around the diagonal so that two of its faces will be perpendicular to the plane of the front-view. We will receive the figure on the left. The thick lines in the figure determine the solid of revolution. It consists of two cones of rotation, one on the top and one at the bottom, and some solid between them.

We do not know what those who had posed the task thought of, they probably did not realize what this solid is. Since it is determined by a line that is skew to the diagonal, it can be neither a cylinder nor a cone, thus it cannot be even a frustum of a cone, and integral calculus was not on the syllabus in secondary schools. To determine the outline of this object, we consider a point $P$ on the edge of the cube with its top-view $P^{\prime}$ and front-view $P^{\prime \prime}$. Let us rotate this point around the diagonal so that the plane of the point and the axis of rotation will be parallel to the plane of the front-view, then we determine the ordinary point of the meridian section of the solid. After rotating $P$, we obtain point $\bar{P}$ (see middle figure).

We determine a parameter representation of the meridian. As a result we will receive the surface generated by any line $g$ skew to the axis of rotation. Let the line that is being rotated have a distance $d$ from the axis of rotation (this can be seen in the top-view), and let it intersect the axis of rotation at an angle $\varphi$. We can see this angle in actual size in the front-view.

Let us introduce a Cartesian co-ordinate frame in the front-view such that $P \in g$ is described by

$$
\begin{aligned}
& y=d \operatorname{tg} \alpha \\
& x=d \operatorname{tg} \alpha \operatorname{ctg} \varphi .
\end{aligned}
$$

The path of the motion can be seen in the top-view.

Accordingly, we obtain for the y co-ordinate of point $\bar{P}$, i.e. the radius of the rotation

$$
y^{2}=d^{2}+d^{2} \operatorname{tg}^{2} \alpha .
$$

We express $\operatorname{tg} \alpha$ from a previous equation,

$$
\operatorname{tg} \alpha=\frac{x}{d \operatorname{ctg} \varphi}
$$


and we substitute this into the former equation after raising it to the second power

$$
y^{2}=d^{2}+d^{2} \operatorname{tg}^{2} \alpha=d^{2}+d^{2} \frac{x^{2}}{d^{2} \operatorname{ctg}^{2} \varphi} .
$$

After reducing this equation, we have

$$
\frac{y^{2}}{d^{2}}-\frac{x^{2}}{d^{2} \operatorname{ctg}^{2} \varphi}=1
$$

and this is the equation of the meridian section. Thus the solid of revolution between the cones is a hyperboloid of one sheet (hyperbolic hyperboloid).

It is obvious from the figure that the altitude of the cones is $\sqrt{3} / 3$. The radius of the base circle of the cones is that of the circle circumscribed around the top-view whose square can be determined with the help of the front-view (the contour is a rectangle).

$$
\rho^{2}=1-\left(\frac{\sqrt{3}}{3}\right)^{2}=1-\frac{3}{9}=\frac{2}{3} \text { (Pythagorean Theorem). }
$$

Thus the sum of the volume of the cones is: $V_{\text {cone }}=\frac{2}{3} \pi \frac{\sqrt{3}}{3} \frac{2}{3}=0.806$ cubic units.

The volume of the hyperboloid can be calculated by integration. In the given problem $\varphi$ and $d$ are special values: $\operatorname{ctg} \varphi=\frac{1}{\sqrt{2}} \rightarrow \operatorname{ctg}^{2} \varphi=\frac{1}{2}, d^{2}=0.5$. We have to integrate from 0 to $\frac{\sqrt{3}}{6}$ according to the formula of the volume of solids of revolution and then multiply it by two.

Thus we have

$$
\begin{aligned}
V_{\text {hyp }} & =2 \pi \int_{0}^{\frac{\sqrt{3}}{6}}\left(d^{2}+2 x^{2}\right) d x=2 \pi \int_{0}^{\frac{\sqrt{3}}{6}}\left(0.5+2 x^{2}\right) d x=2 \pi\left[0.5 x+2 \frac{x^{3}}{3}\right]_{0}^{\frac{\sqrt{3}}{6}} \\
& =\pi\left(\frac{\sqrt{3}}{6}+\frac{4 \cdot 3 \sqrt{3}}{3 \cdot 6^{3}}\right) \\
V_{\text {hyp }} & =1.007 .
\end{aligned}
$$

The answer to the question is $V=0.806+1.007=1.813$ cubic units.

The figure on the right shows the top- and front-view of the solid of the revolution.

We think that the way the surface of rotation generated by a line skew to the axis is simple enough to be taught even at secondary school level: According to the relative situation of the line being rotated and the axis of rotation the surface of revolution can be a cone, a cylinder or a one sheet hyperboloid. 
The following task was worth the most points in a competition in the school year $1973-74$ in the first round [5, page 22].

Problem 7. A two-dimensional abstract animal is wandering on a unit sphere. The length of its steps is 1.9 measured on a straight line (not on an arc). How many steps has the animal to take at least to return to its startingpoint? ("Wandering" implies that it cannot turn back there immediately after the first step.)

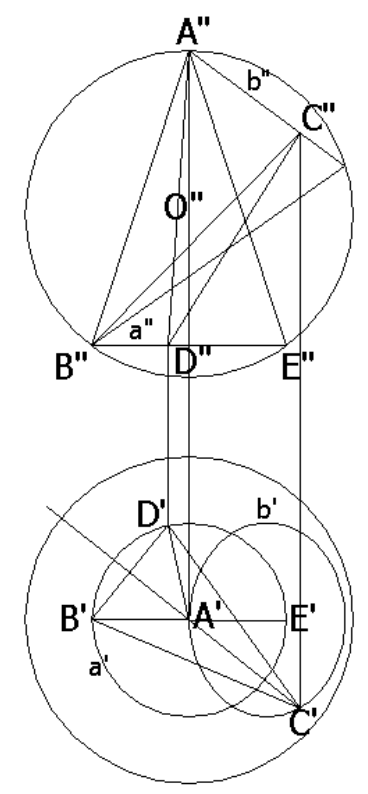

Solution. 1. Three steps are insufficient because the radius of the circle circumscribed around the equilateral triangle of sides 1.9 is $r=\frac{1.9}{2} * \sqrt{3} * \frac{2}{3} \approx$ 1.096965 units, so there don't exist any circles of this radius on the unit sphere.

2. Four steps are enough: We construct four points $A, B, C, D$ on the sphere, whereby consecutive pairs of points $A-B-C-D-A$ have 1.9 units as distance. We start with the top- and front-view and put $A$ at the 'north pole' and $O$ in the center of the sphere. The set of points on the sphere that are at a distance of 1.9 from $A$ is a circle $a$ with $A O$ as its axis of rotation. Choose one of its points denoted by $B$. Now the animal can step from here to a point on the 


$$
\text { "szabo 'molnar" — 2004/7/22 — 15:23 — page } 115 \text { — \#13 }
$$

circle $b$ passing through $A$ opposite to $B$. The top-view of circle $b$ is an ellipse and its front-view is a segment. Now $D$ has to lay on $a$, because the distance of $A$ and $D$ has to be 1.9. Since $d(C B)=d(C D)(=1.9)$, the point $C$ lays within the symmetry plane of segment $B D$. This symmetry plane is projecting in the top-view projection, because $B D$ is parallel to the first image-plane, and it passes through $A$ and intersects $b$ in a second point $C$. So to any chosen point $D(D \neq B$ and $D \neq E$ where $B E$ is a diameter of $a)$ there exists a point $C \in b$ and the quadrangle $A B C D$ solves the problem.

\section{References}

[1] J. Horváth, Anwendungen der Projektionmethode im Unterricht und in der Forschung, Symposium Darstellende Geometrie, Dresden, 2000, Proceedings, S 47-52.

[2] F. Kárteszi, Ábrázoló geometria, Tankönyvkiadó, Budapest, 1962.

[3] Középiskolai matematikai versenyek 1980-1984, Tankönyvkiadó, Budapest, 1986, Osszeállította Fried Katalin és Pogáts Ferenc.

[4] E. Müller, Vorlesungen über Darstellenden Geometrie, II., Die Zyklographie von J. L. Krames, Wien, 1928.

[5] Középiskolai matematikai versenyek 1973/74, Tankönykiadó, Budapest, 1977.

[6] Skljarszkij - Csencov - Jaglom, Válogatott feladatok és tételek az elemi matematika köréből, Sztereometria, Tankönyvkiadó, Budapest, 1968.

[7] E. Stiefel, Lehrbuch der Darstellenden Geometrie, 3., Aufl. Basel, Stutgart, 1971.

[8] L. Vigassy, Síkmértani szerkesztések térmértani megoldással, Középiskolai szakköri füzetek, Tankönyvkiadó, Budapest, 1975.

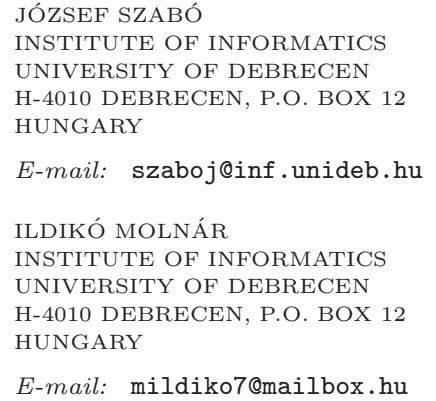

(Received February, 2004) 